\section{Expression and function of cathelicidin hCAP18/LL-37 in chronic lymphocytic leukemia}

Chronic lymphocytic leukemia (CLL) is characterized by the accumulation of clonal B-cells in peripheral blood and lymphoid tissues. ${ }^{1}$ Circulating CLL cells are nondividing B lymphocytes, but a significant fraction of the clone proliferates in lymphoid tissues where they receive a plethora of signals from the microenvironment that promote their survival and expansion. ${ }^{2}$ Cathelicidins are a family of proteins with antibacterial functions mainly expressed by neutrophils, macrophages and epithelial cells. ${ }^{3}$ In humans, the only member of this family, hCAP18, is encoded by the gene CAMP. The cleavage of hCAP18 generates the antimicrobial peptide LL-37, which has been recently implicated in the promotion of tumor growth, through direct stimulation of malignant cells, initiation of angiogenesis and recruitment of immune cells. ${ }^{4}$ In this study, we investigated the role of hCAP18/LL-37 in CLL.

Clinical features of analyzed patients are shown in the Online Supplementary Table S1. By gene expression analysis, we found that CAMP is up-regulated in CLL cells compared to B cells from age-matched healthy donors (HD) (Online Supplementary Figure S1), and this was confirmed by qRT-PCR (Figure 1A). Interestingly, when samples were discriminated according to their immunoglobulin heavy chain variable region (IGHV) mutation status, those with unmutated IGHV (poor clinical outcome, UCLL) showed significantly higher levels than those with mutated IGHV (better outcome, M-CLL). Similarly, leukemic cells from patients in Rai III/IV stages expressed higher levels of $h C A P 18$ mRNA compared to indolent Rai 0 patients (Figure 1B), suggesting that transcription of CAMP is related to an aggressive clinical phenotype. Nevertheless, flow cytometry analysis, using polymorphonuclear cells (PMN) as a positive control, did not reveal hCAP-18/LL-37 protein expression in circulating CLL cells or B lymphocytes from healthy donors (Online Supplementary Figure S2).

Given that CLL cells in lymphoid tissues possess a distinct phenotype, with enhanced expression of anti-apoptotic and activation markers compared to circulating cells; ${ }^{5}$ we looked for hCAP18/LL-37 in bone marrow biopsies. Figure $1 \mathrm{C}$ shows that a proportion of leukemic cells $\left(\mathrm{CD} 20^{+}\right)$and almost all myeloid cells $\left(\mathrm{CD}^{+} 8^{+}\right)$cells expressed hCAP18/LL-37. These results suggest that signals from the microenvironment promote the expression of hCAP-18/LL-37 protein in CLL cells. To assess this possibility we stimulated circulating CLL cells with different well-known stimuli, such as CD40L, CpG, immobilized anti-IgM, interleukin (IL)-4 (IL-4) and IL-15. Results show that these stimuli up-regulated the transcription of CAMP (Figure 1D) and, more importantly, those stimuli that were most effective in increasing $h$ CAP 18 mRNA, i.e., $\mathrm{CpG}^{+} \mathrm{IL} 15$ and $\mathrm{CD} 40 \mathrm{~L}^{+} \mathrm{IL} 4$, induce the expression of hCAP18/LL-37 protein (Figure 1E). We also observed a significant though moderate correlation between mRNA and protein levels of hCAP18/LL-37 after cellular activation (Online Supplementary Figure S3). Compared to neutrophils, the intracellular expression of hCAP18/LL-37 in activated CLL was modest. However it should be taken into account that hCAP18/LL-37 is stored in neutrophils granules at very high concentrations $\left(0.6 \mu \mathrm{g} / 10^{6}\right.$ cells $)$, while it appears to be released to supernatant by activated CLL cells (Figure 1F).

hCAP18/LL-37 has been shown to participate as a tumor promoting factor in breast and lung cancers and in pancreatic ductal adenocarcinoma. ${ }^{4,7}$ In those settings, hCAP18/LL-37 acts mainly as a growth factor inducing tumor cell activation, survival and proliferation. Since CLL cells proliferate minimally in vitro, even upon strong stimulation, we assessed if LL-37 could induce leukemic cell activation and inhibit spontaneous and drug-induced apoptosis. We found that incubation of CLL cells with LL-37 at 5 or $10 \mu \mathrm{M}$ did not increase the expression of activation markers (not shown), but significantly delayed spontaneous apoptosis of leukemic cells as evaluated by binding of Annexin V (Figure 2A-B). These concentrations are found at sites of inflammation, like bronchoalveolar lavage fluid from infants with pulmonary infections $s^{8}$ or psoriatic skin lesions. ${ }^{9}$ Of note, LL-37 not only inhibited spontaneous CLL apoptosis but also interfered with that induced by ABT-199 or fludarabine, agents currently employed for CLL treatment ${ }^{1}$ (Figure 2C$\mathrm{D}$ and Online Supplementary Figures 4-5). In addition to AnnexinV staining, inhibition of apoptosis by LL-37 was corroborated analyzing caspase 3 cleavage (Figure 2E) and BCL-2 expression (Online Supplementary Figure S5B). Because delay of neutrophil apoptosis by LL-37 has been reported to depend on two different receptors: FPRL1 and P2X7 $7,{ }^{10}$ we tested if WRW4 (FPRL1 antagonist) or $\mathrm{KN}-62$ (P2X7 inhibitor) could counteract the protective effect of LL-37. Figure 3A shows that none of these agents interfered with LL-37 activity indicating that neither FPRL1 nor P2X7 were involved.

LL-37 can also interact with CXCR $4,{ }^{11}$ the receptor for the chemokine CXCL12, which plays an important role in CLL by promoting the migration of leukemic cells to lymphoid tissues and improving their survival. ${ }^{12}$ Therefore, we determined if CXCR4 was involved in the anti-apoptotic pathway triggered by LL-37. We found that an antibody directed to CXCR4 that blocks CXCL12 engagement impaired LL-37 protection (Figure 3B). Furthermore, confocal microscopy shows co-localization of LL-37 and CXCR4 on the cell membrane indicating that both molecules are in the same patches on CLL surface (Figure 3C). Finally, we evaluated CXCR4 endocytosis since this is a mandatory step for chemokine receptors upon binding cognate chemokine, in this case CXCL12. The interaction of CXCR4 with LL-37 at $37^{\circ} \mathrm{C}$ led to downregulation of CXCR4 from the CLL cell surface (Figure 3D). Altogether, these results suggest that CXCR4 could be acting as a LL-37 receptor on CLL cells.

Since it has previously been reported that LL-37 enhances the chemotactic responsiveness of hematopoietic progenitors to low doses of CXCL12, ${ }^{11}$ we evaluated if this was also true for CLL cells. To address this, we used transwell chambers and assessed leukemic cell migration towards a suboptimal concentration of CXCL12 $(25 \mathrm{ng} / \mathrm{mL})$, with or without LL-37 (5 $\mu \mathrm{M})$. As was the case for hematopoietic progenitors, LL-37 increased the migratory response of CLL cells to CXCL12. Of note, LL-37 did not induce chemotaxis of CLL cells by itself, as demonstrated in neutrophils acting through FPRL $1^{10}$ and in keratinocytes through transactivation of the epidermal growth factor receptor ${ }^{13}$ (Figure 3E).

Accumulating evidence supports pro- or anti-tumorigenic roles for hCAP18/LL-37 in various types of human cancer, e.g., pro-tumor in ovarian, lung and breast cancer and in malignant melanoma, and anti-tumor in colon and gastric tumors. ${ }^{4}$ In agreement with its inflammatory and pro-angiogenic activity, hCAP-18/LL-37 expression in most solid tumors correlates with increased leukocyte infiltration and microvessel density. In breast and lung 
A

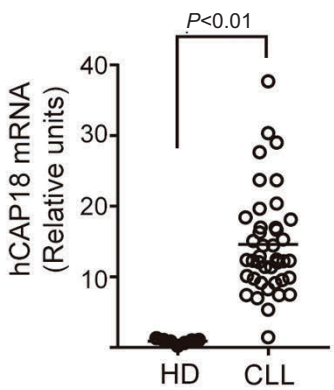

B

Low hCAP18 High hCAP18
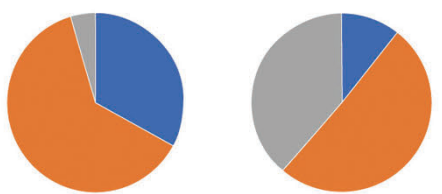

$0 \quad \square \mathrm{I} / \mathrm{II} \square \mathrm{III} / \mathrm{IV}$

RAl stage

C
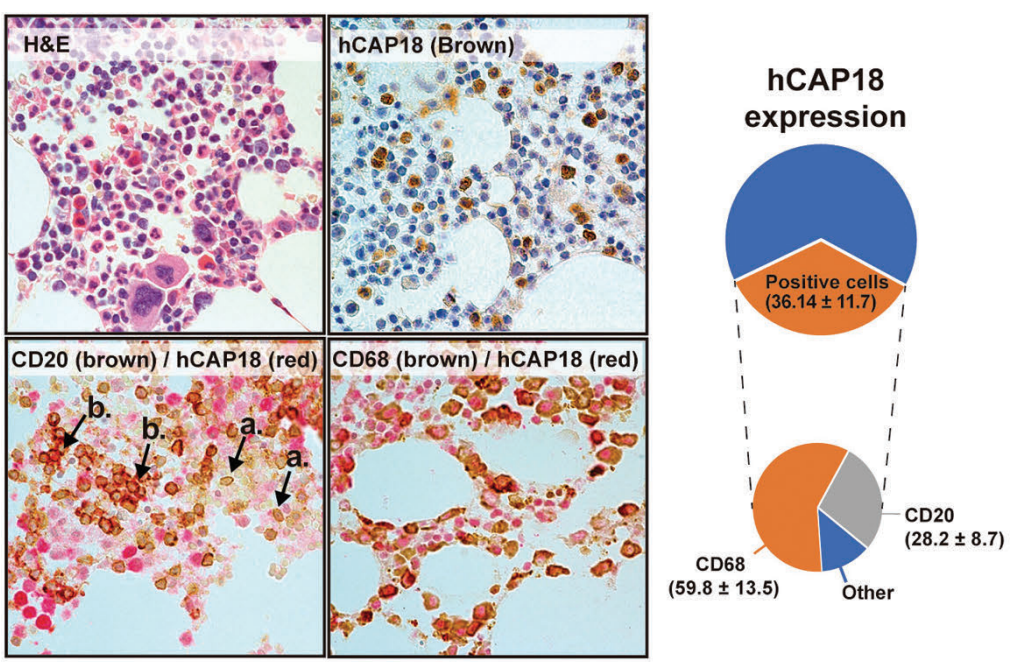

D

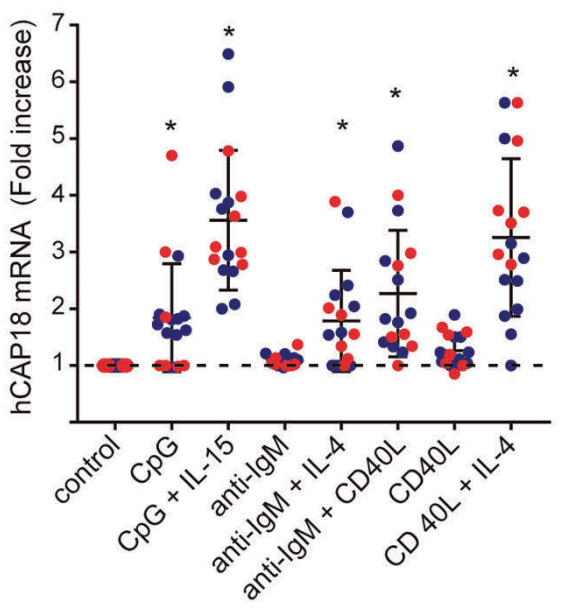

E

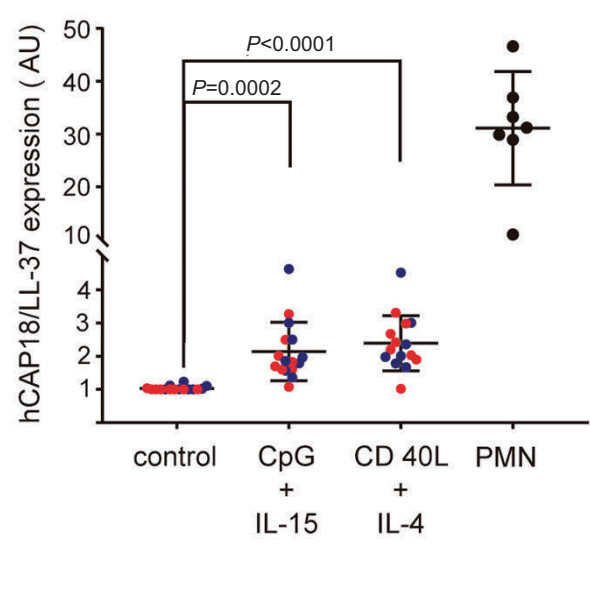

F

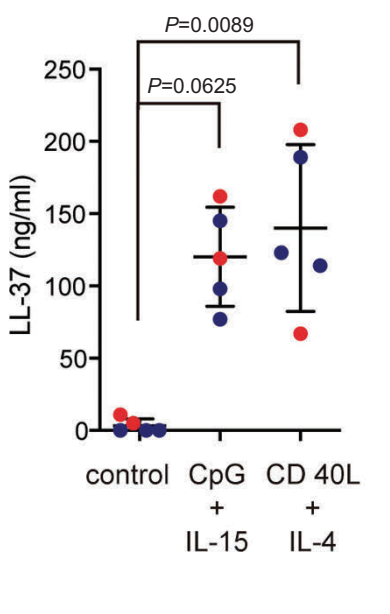

Figure 1. hCAP18/LL-37 expression in chronic lymphocytic leukemia cells. (A) Left panel: hCAP18 mRNA expression was evaluated in purified B-cells from healthy donors (HD) $(n=7)$ and chronic lymphocytic leukemia (CLL) cells $(n=41)$ samples by qRT-PCR. Results were normalized to $\beta$-actin human gene and are represented as relative units $\left(2-\Delta \mathrm{ct} \times 10^{3}\right)$. Statistical analysis was performed using Mann-Whitney test. Shown are individual values and mean \pm standard error of the mean (SEM). Right panel: hCAP18 mRNA expression on CLL cell samples discriminated based on their immunoglobulin heavy chain variable region (IGHV) mutational status. Unmutated (U-CLL) $n=21$, Mutated (M-CLL) $n=20$. (B) The average expression of mRNA $h C A P 18$ in CLL cell samples was calculated and

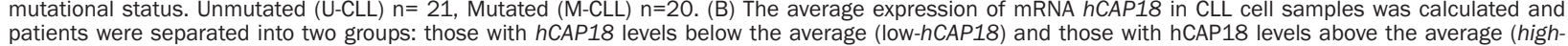
$h C A P 18) \cdot \chi^{2}$ test showed a statistically significant association between hCAP18 expression and CLL clinical stage (Rai staging). (C) Expression of hCAP18/LL-37 on bone marrow biopsies from CLL patients. Samples were doubled-stained to identify LL-37 in myeloid cells (CD68 ${ }^{+}$) and CLL cells (CD20 ${ }^{+}$) as detailed in the Online Supplementary Materials and Methods. Shown are representative pictures for haematoxylin and eosin (H/E) staining, hCAP18 single staining and CD68 ${ }^{+}$ or $\mathrm{CD} 20^{+} / \mathrm{hCAP} 18$ double staining, $\mathrm{n}=5$ (left panel). Image magnification: $600 \mathrm{x}$. Arrow heads represent hCAP18 single positive cells (a) or double positive (CD20/hCAP18) cells (b). In the right panel, pie charts displaying the total percentage of hCAP18 positive cells and the contribution of each cell type (CD68 ${ }^{+}, \mathrm{CD} 20^{+}$and others) are shown (D) hCAP18 mRNA expression in CLL cells stimulated with CpG (5 $\left.\mathrm{\mu g} / \mathrm{mL}\right), \mathrm{CpG}+\mathrm{IL}-15$ (10 ng/mL), immobilized anti-lgM antibody $(\mathrm{Ab})(0.1 \mathrm{\mu g} / \mathrm{mL})$, anti-lgM Ab $+\mathrm{IL}-4(15 \mathrm{ng} / \mathrm{mL})$, anti-lgM Ab $+\mathrm{CD} 40 \mathrm{~L}(500 \mathrm{ng} / \mathrm{mL})$, CD40L or CD4OL $+\mathrm{IL}-4 \mathrm{for} 48 \mathrm{hours}(\mathrm{h})$ at $37^{\circ} \mathrm{C}$. Results are expressed as fold increase compared to control (unstimulated condition). Statistical analysis was performed using Friedman test and Dunn's multiple comparison test. Shown are individual values and mean \pm standard error of the mean (SEM). Blue dots correspond to M-CLL and red dots to U-CLL samples. Asterisks represent $P<0.05$. E. Intracellular expression of hCAP18/LL-37 in CLL cells activated with CpG + IL-15 or CD40L + IL-4 for $48 \mathrm{~h}$ at $37^{\circ} \mathrm{C}$. Results are expressed as the ratio of median fluorescence intensity (MFI) of hCAP18/LL-37 to MFI of isotype control in CD19+ cells (blue dots: M-CLL, red dots: U-CLL) or in PMN (positive control). Individual values and mean + SEM are shown. F. LL-37 concentration in culture supernatants of activated CLL-cells quantified by enzyme linked immunabsorbant assay (ELISA). Statistical analysis was performed using Friedman test and Dunn's multiple comparison test $(P<0.05)$. Shown are individual values and mean \pm SEM. (blue dots: M-CLL, red dots: U-CLL). 
A

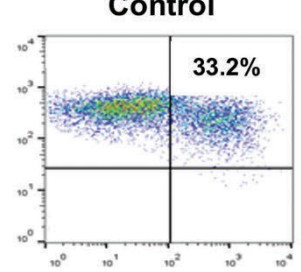

LL-37 5 $\mu \mathrm{m}$

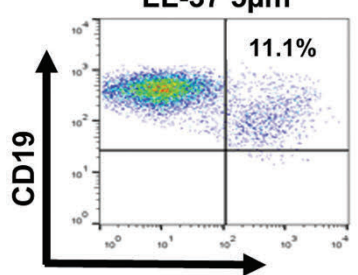

Annexin V

C

Control

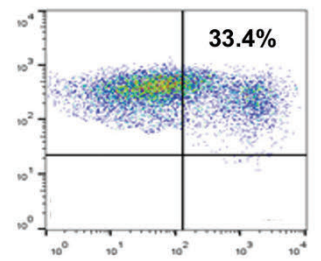

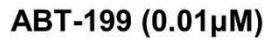

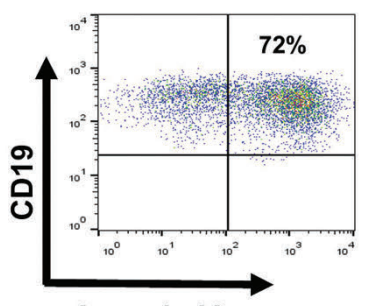

Annexin V

E

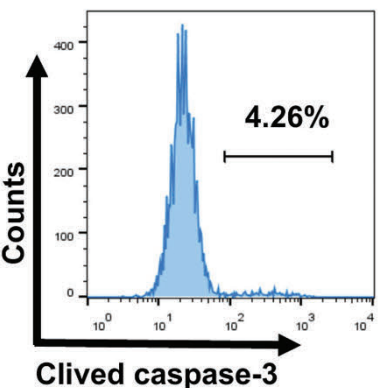

LL-37 2.5 $\mu \mathrm{m}$

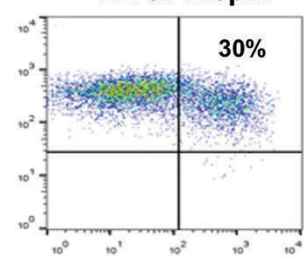

LL-37 10 $\mu \mathrm{m}$

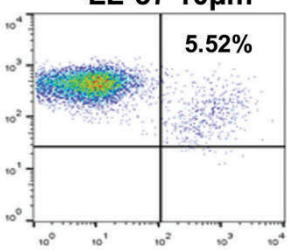

LL-37 5 $\mu \mathrm{m}$

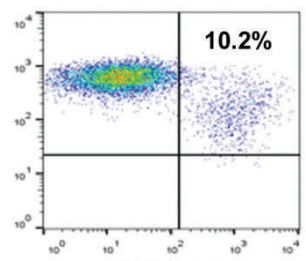

ABT-199 + LL-37

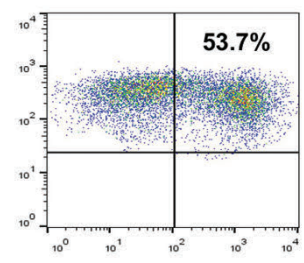

$\mathrm{ABT}-199(0.01 \mu \mathrm{M})$

D
B
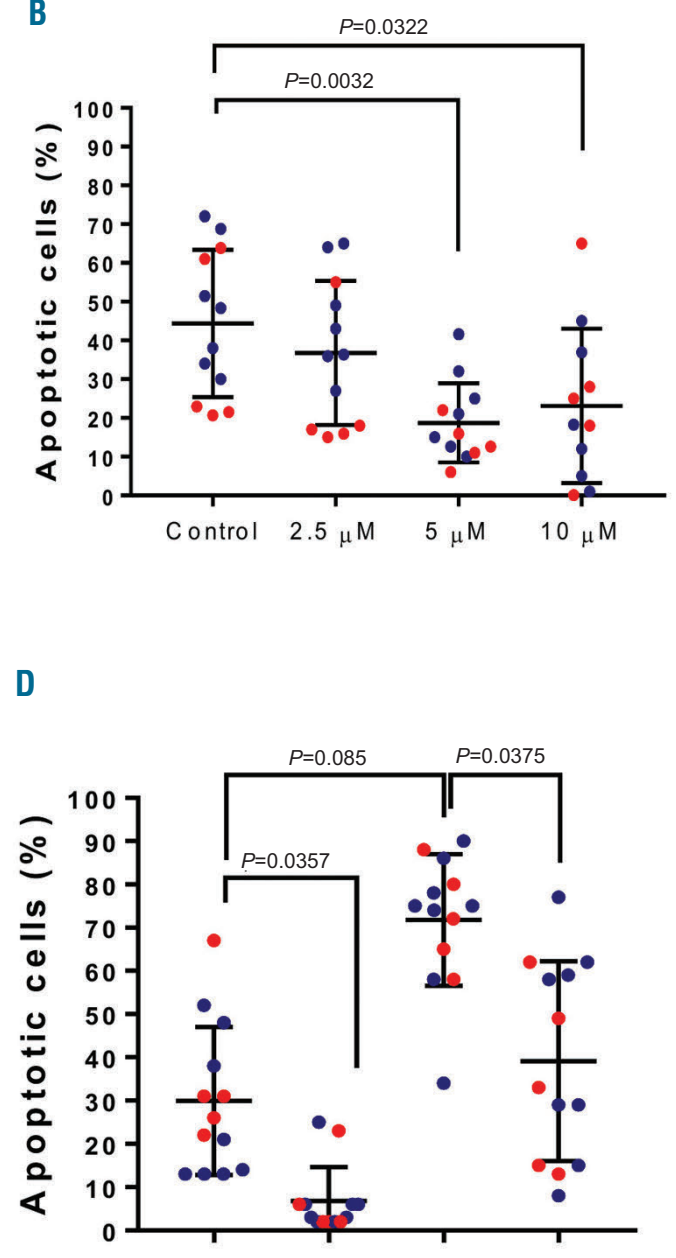

$\mathrm{LL}-37(5 \mu \mathrm{M}) \quad-\quad+\quad+$

Figure 2. LL-37 inhibits spontaneous and ABT-199 induced apoptosis of chronic lymphocytic leukemia cells. Peripheral blood mononuclear cells (PBMC) samples from chronic lymphocytic leukemia (CLL) patients were incubated with LL-37 (2.5-10 $\mu \mathrm{M})$ for 48 hours (h) $37^{\circ} \mathrm{C}$. Cells were stained with PC5-anti-CD19 mAb and AnnexinV-FITC and analyzed by flow cytometry. (A) Shown are representative CD19 versus AnnexinV dot plots (blue dots: M-CLL, red dots: U-CLL) and the percentage of apoptotic cells (mean \pm standard error of the mean [SEM], $n=12$ ). (B) PBMC samples from CLL patients were incubated with ABT-199 (0.01 $\mu \mathrm{M})$ with or without LL-37 (5 $\mu \mathrm{M})$ for $48 \mathrm{~h}$ at $37^{\circ} \mathrm{C}$. (C-D) Apoptosis was assessed by flow cytometry as described in (A). Depicted are representative CD19 versus AnnexinV dot plots and the percentage of apoptotic cells (mean \pm standard error of the mean [SEM], $n=13$ ). Statistical analysis was performed using Friedman test and Dunn's multiple comparison test $(P<0.05)$. (E) Leukemic cells were treated with ABT-199 $(0.1 \mu \mathrm{M})$ for $18 \mathrm{~h}$ with or without LL-37 (5 $\mu \mathrm{M})$. The activation of the apoptotic program was analyzed by caspase-3 cleavage and flow cytometry. Shown are representative histograms ( $n=5)$. 
A
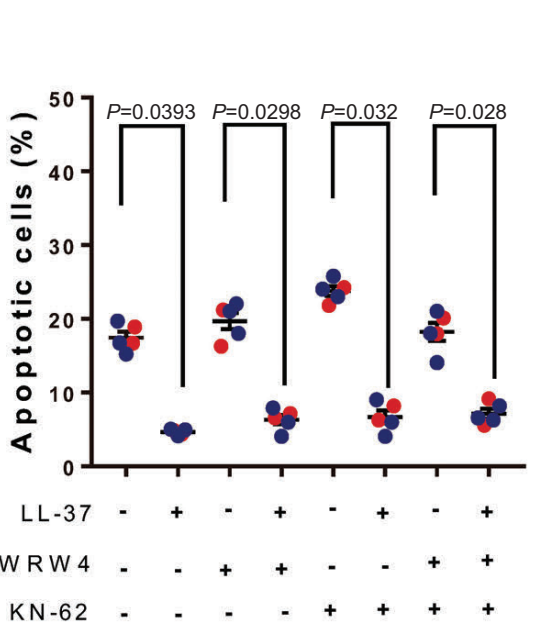

LL-37

C

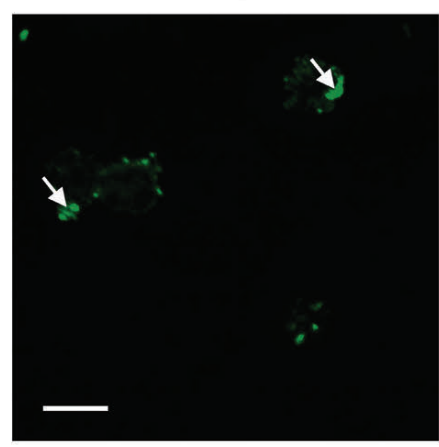

B

24h

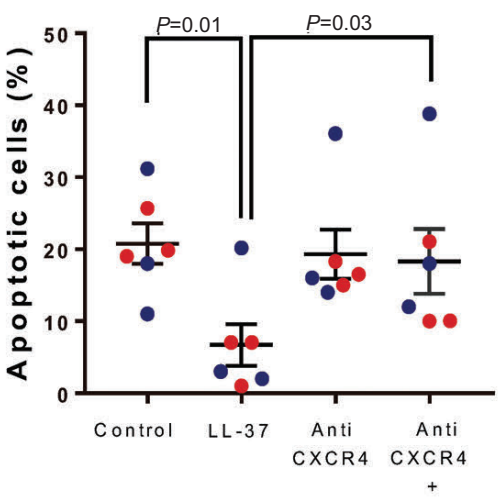

LL-37 48h

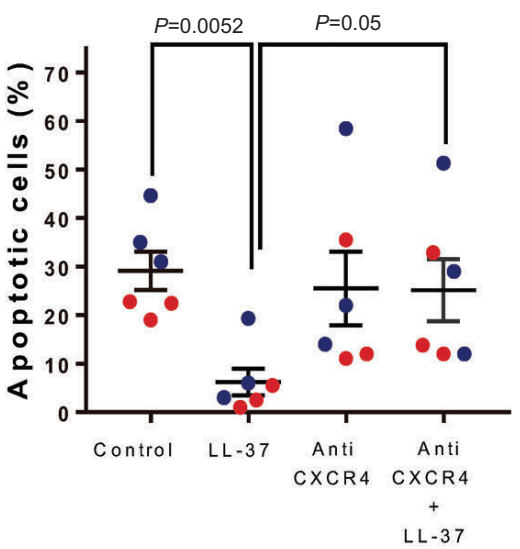

CXCR4

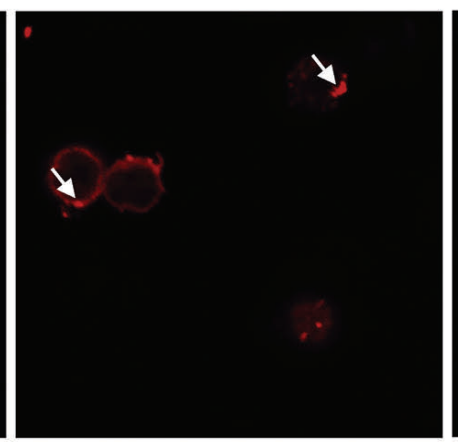

Merge

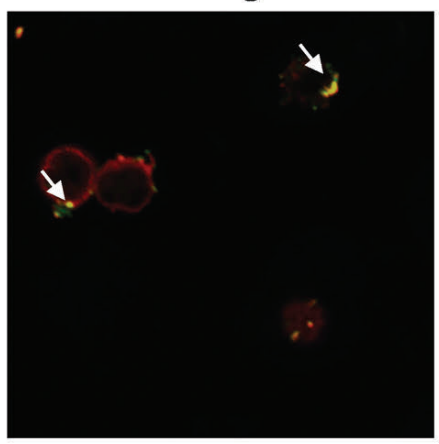

E

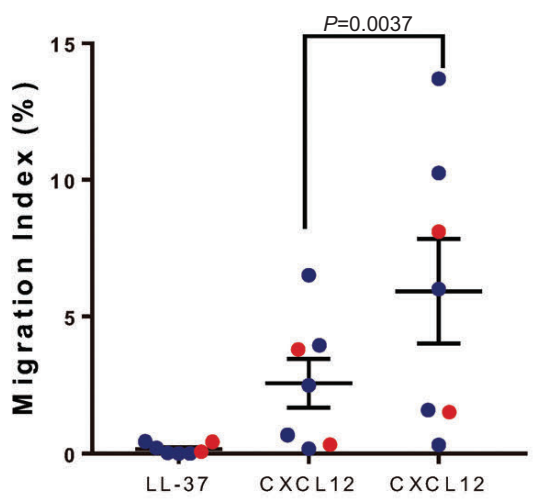

LL -37

Figure 3. Role of CXCR4 in LL-37 effects on chronic lymphocytic leukemia cells. Peripheral blood mononuclear cells (PBMC) from chronic lymphocytic leukemia (CLL) patients were incubated with WRW4 $(1 \mu \mathrm{M}), \mathrm{KN}-62(1 \mu \mathrm{M})$ or both for 30 min before adding LL-37 (5 $\mu \mathrm{M})$. Cells were cultured for 48 hours $(\mathrm{h})$ at $37^{\circ} \mathrm{C}$ and apoptosis was evaluated as described in Figure 2. (A) The percentages of apoptotic CLL cells are shown (mean \pm standard error of the mean [SEM], $n=5$ ). Statistical analysis was performed using Friedman test and Dunn's multiple comparison test $(P<0.05)$. (B) PBMC from CLL patients were incubated with antiCXCR4 Ab $(10 \mu \mathrm{g} / \mathrm{mL})$ for 30 min before adding LL-37 $5 \mu \mathrm{M}$. Cells were cultured for $48 \mathrm{~h}$ at $37^{\circ} \mathrm{C}$. A fresh aliquot of anti-CXCR4 was re-added at $24 \mathrm{~h}$. Apoptosis was evaluated at 24 and $48 \mathrm{~h}$ as described in Figure 2. Shown are the percentages of apoptotic cells (mean \pm SEM, $n=6$ ). Statistical analysis was performed using Friedman test and Dunn's multiple comparison test $(P<0.05)$. (C) CLL cells were incubated for 30 min with LL-37 (5 $\mu$ M), then fixed with $4 \%$ paraformaldehyde and labeled with rabbit anti-LL-37 IgG followed by Dy-Light 488-anti-rabbit IgG (green) and anti-CXCR4-PE IgG (red). Colocalization areas are indicated by the arrowheads. The bar indicates 15 m. (D) Time-dependent downregulation of CXCR4 on CLL cells induced by LL-37. CLL cells were incubated with LL-37 $(5 \mathrm{uM})$ at $37^{\circ} \mathrm{C}$ for the indicated time and the expression of membrane CXCR4 was evaluated by flow cytometry. Results are expressed as mean flouresence intensisty (MFI) (mean \pm SEM, $n=5)$. Statistical analysis was performed using Friedman test and Dunn's multiple comparison test $(P<0.05)$. ( $E$ ) LL-37 enhances the migration of LLC cells towards CXCL12. CLL cells $\left(2 \times 10^{6}\right.$ cells $\left./ \mathrm{mL}\right)$ were seeded in the upper chamber of transwell plates to evaluate their migration to the lower compartment in response to CXCL12 $(25 \mathrm{ng} / \mathrm{mL})$ in the presence or absence of LL-37 (5 uM). Cells were incubated for $120 \mathrm{~min}$, recovered from the lower chamber and quantified by flow cytometry. The migration index was calculated as the subtraction of the number of CD $19^{+}$cells that migrated spontaneously (control without chemokine) from the number of CD19+ cells that migrated in the presence of CXCL12 and normalized to the input. Shown are mean \pm SEM $(n=7)$. Statistical analysis was performed using Friedman test and Dunn's multiple comparison test $(P<0.05)$. In all cases red dots represent $U$-CLL and blue dots M-CLL. 
cancers, exogenous LL-37 promotes cell proliferation and metastasis, ${ }^{4}$ but it induces G0/G1 cell cycle arrest in gastric cancer cells, suggesting that the effect of hCAP18/LL37 depends on the tissue origin of the tumor. Moreover, it is important to consider that hCAP18/LL-37 can also be expressed by stromal and myeloid cells in the microenvironment. In fact, malignant cells are able to promote the expression of hCAP18/LL-37 by tumor associated macrophages as occurs in pancreatic ductal adenocarcinomas where the secreted peptide contributes to tumor progression. ${ }^{7}$ Similarly, in a murine model of prostatic cancer, overexpression of CAMP in tumor cells favors the differentiation of early myeloid progenitors into protumorigenic M2 macrophages. ${ }^{14}$

Here we show that, unlike normal B lymphocytes, leukemic B cells from CLL patients express $h C A P 18$ mRNA and that this expression associates with poor prognosis and disease stage. Interestingly, we found that circulating CLL cells can produce and release hCAP18/LL-37 protein upon in vitro activation with microenvironmental stimuli and this observation was supported by bone marrow immunohistochemistry. In vitro, exogenous LL-37 delays CLL cell apoptosis, both spontaneous and apoptosis induced by chemotherapeutic agents. As an antimicrobial peptide, the main function of LL-37 is to induce bacterial lysis via the formation of transmembrane pores. ${ }^{15}$ Cellular membranes associated with cholesterol, such as those from human cells, are resistant to LL-37 toxicity unless supraphysiological concentrations are used. By contrast, LL-37 has antiapoptotic effects in a variety of cells including neutrophils through FPRL1 and P2X7.${ }^{10}$ We have shown that these two receptors are not involved in CLL cell protection by LL-37, but rather this depends on CXCR4, a key receptor for CLL cell survival and traffic. Of note, LL-37 was able to enhance leukemic cell migration towards suboptimal concentrations of CXCL12.

In conclusion, our results suggest that hCAP18/LL-37 may have an active role in the CLL-tumor microenvironment by increasing leukemic clone retention and survival in lymphoid tissues.

Enrique Podaza, ${ }^{1}$ Florencia Palacios, ${ }^{2}$ Diego O. Croci, ${ }^{3}$ Denise Risnik, ${ }^{1}$ Xiao J. Yan, ${ }^{2}$ María Belén Almejún, ${ }^{1}$ Ana Colado, ${ }^{1}$ Esteban E. Elias, ${ }^{1}$ Mercedes Borge, Pablo E. Morande, ${ }^{1}$ Raimundo Bezares, ${ }^{4}$

Horacio Fernández-Grecco, Gabriel A. Rabinovich, ${ }^{6}$ Romina Gamberale, ${ }^{1}$ Nicholas Chiorazzi ${ }^{2}$ and Mirta Giordano ${ }^{1}$

'Laboratorio de Inmunología Oncológica, Instituto de Medicina Experimental/CONICET, Buenos Aires, Argentina; ${ }^{2}$ Karches Center for Oncology Research, The Feinstein Institute for Medical Research, Manhasset, NY, USA; ${ }^{3}$ Laboratorio de Inmunopatologia, Instituto de Histología y Embriología de Mendoza/CONICET, Facultad de Ciencias Exactas y Naturales, UNC, Mendoza, Argentina; ${ }^{4}$ Servicio de Hematología, Hospital General de Agudos, Dr. Teodoro Álvarez, Buenos Aires, Argentina; SServicio de Hematología, Sanatorio Municipal Dr. Julio Méndez, Buenos Aires, Argentina and 'Laboratorio de Inmunopatología, Instituto de Biología y Medicina Experimental/ CONICET and Facultad de Ciencias Exactas y Naturales, Universidad de Buenos Aires, Buenos Aires, Argentina
Correspondence:

MIRTA GIORDANO - giordanomirta@gmail.com

doi:10.3324/haematol.2019.227975

Acknowledgments: the authors would like to thank Maria Tejeda, Romina Pagano and Federico Fuentes for technical assistance. We are grateful for the participation of the patients and control subjects in this study.

Funding: this work was supported by grants from Agencia Nacional de Promoción Cientifica y Tecnológica, Argentina.

\section{References}

1. Hallek M, Shanafelt TD, Eichhorst B. Chronic lymphocytic leukaemia. Lancet. 2018;391(10129):1524-1537.

2. Herndon TM, Chen SS, Saba NS, et al. Direct in vivo evidence for increased proliferation of CLL cells in lymph nodes compared to bone marrow and peripheral blood. Leukemia. 2017;31(6):13401347.

3. Kahlenberg JM, Kaplan MJ. Little peptide, big effects: the role of LL37 in inflammation and autoimmune disease. J Immunol. 2013;191(10):4895-4901.

4. Wu WK, Wang G, Coffelt SB, et al. Emerging roles of the host defense peptide LL-37 in human cancer and its potential therapeutic applications. Int J Cancer. 2010;127(8):1741-1747.

5. Herishanu Y, Perez-Galan P, Liu D, et al. The lymph node microenvironment promotes B-cell receptor signaling, NF-kappaB activation, and tumor proliferation in chronic lymphocytic leukemia. Blood. 2011;117(2):563-574.

6. Sorensen O, Cowland JB, Askaa J, Borregaard N. An ELISA for hCAP-18, the cathelicidin present in human neutrophils and plasma. J Immunol Methods. 1997;206(1-2):53-59.

7. Sainz B, Jr., Alcala S, Garcia E, et al. Microenvironmental hCAP18/LL-37 promotes pancreatic ductal adenocarcinoma by activating its cancer stem cell compartment. Gut. 2015;64(12):1921-1935.

8. Schaller-Bals S, Schulze A, Bals R. Increased levels of antimicrobial peptides in tracheal aspirates of newborn infants during infection. Am J Respir Crit Care Med. 2002;165(7):992-995.

9. Ong PY, Ohtake T, Brandt C, et al. Endogenous antimicrobial peptides and skin infections in atopic dermatitis. $N$ Engl J Med. 2002;347(15):1151-1160.

10. Nagaoka I, Tamura H, Hirata M. An antimicrobial cathelicidin peptide, human CAP18/LL-37, suppresses neutrophil apoptosis via the activation of formyl-peptide receptor-like 1 and P2X7. J Immunol. 2006;176(5):3044-3052.

11. Wu W, Kim CH, Liu R, et al. The bone marrow-expressed antimicrobial cationic peptide LL-37 enhances the responsiveness of hematopoietic stem progenitor cells to an SDF-1 gradient and accelerates their engraftment after transplantation. Leukemia. 2012;26(4):736-745

12. Burger JA, Montserrat E. Coming full circle: 70 years of chronic lymphocytic leukemia cell redistribution, from glucocorticoids to inhibitors of B-cell receptor signaling. Blood. 2013;121(79):15011509.

13. Wang DY, Cheng CC, Kao MH, Hsueh YJ, Ma DH, Chen JK. Regulation of limbal keratinocyte proliferation and differentiation by TAp63 and DeltaNp63 transcription factors. Inv Ophthalmol Vis Sci. 2005;46(9):3102-3108.

14. Cha HR, Lee JH, Hensel JA, et al. Prostate cancer-derived cathelicidinrelated antimicrobial peptide facilitates macrophage differentiation and polarization of immature myeloid progenitors to protumorigenic macrophages. Prostate. 2016;76(7):624-636.

15. Lee CC, Sun Y, Qian S, Huang HW. Transmembrane pores formed by human antimicrobial peptide LL-37. Biophys J. 2011;100(7):16881696. 